\title{
Hepatocyte growth factor and soluble cMet levels in plasma are prognostic biomarkers of mortality in patients with severe acute kidney injury
}

\author{
Lilin $\mathrm{Li}^{1,2}$, Jung Nam An ${ }^{3}$, Jeonghwan Lee ${ }^{1,4}$, Dong Jin Shin ${ }^{5}$, Shi Mao Zhu ${ }^{4}$, Jin Hyuk Kim ${ }^{4}$, Dong Ki Kim ${ }^{6}$, \\ Dong-Ryeol Ryu ${ }^{7}$, Sejoong Kim ${ }^{8}$, Jung Pyo Lee ${ }^{1,4}$
}

${ }^{1}$ Department of Internal Medicine, Seoul National University College of Medicine, Seoul, Republic of Korea

${ }^{2}$ Department of Intensive Care Unit, Yanbian University Hospital, Jilin, China

${ }^{3}$ Department of Internal Medicine, Hallym Sacred Heart Hospital, Anyang, Republic of Korea

${ }^{4}$ Department of Internal Medicine-Nephrology, Seoul National University Boramae Medical Center, Seoul, Republic of Korea

${ }^{5}$ Preliminary Medicine Courses, Seoul National University College of Medicine, Seoul, Republic of Korea

${ }^{6}$ Department of Internal Medicine-Nephrology, Seoul National University Hospital, Seoul, Republic of Korea

${ }^{7}$ Department of Internal Medicine-Nephrology, Ewha Womans University School of Medicine, Seoul, Republic of Korea

${ }^{8}$ Department of Internal Medicine-Nephrology, Seoul National University Bundang Hospital, Republic of Korea

Background: Hepatocyte growth factor (HGF)/cMet pathway is necessary for repair and regeneration following acute kidney injury (AKI). We evaluated the clinical potential of plasma HGF and soluble cMet as prognostic biomarkers for severe AKI requiring continuous renal replacement therapy (CRRT).

Methods: One hundred thirty-six patients with severe AKI who participated in the VENUS (volume management under body composition monitoring in critically ill patients on CRRT) trial between 2017 and 2019 were enrolled in this study. We investigated associations between plasma HGF and cMet concentrations and all-cause mortality.

Results: Plasma HGF and soluble cMet levels were positively correlated. Patients were divided into three groups based on their HGF and soluble cMet concentrations. The day D 0, D2, and D7 highest concentration HGF groups had significantly higher in-hospital mortality after adjusting for sex, body mass index, Acute Physiology and Chronic Health Evaluation II, and age-adjusted Charlson comorbidity index score, especially on D7 (hazard ratio, 4.26; 95\% confidence interval, 1.71-10.62; $p=0.002$ ). D7 soluble cMet level was also associated with mortality. Receiver operating characteristic curve analysis indicated that D7 HGF and soluble cMet levels were best at predicting mortality. Addition of plasma HGF and soluble cMet to conventional prognostic indices significantly improved the predictive value for mortality on D7. However, plasma HGF and soluble cMet were not associated with fluid status.

Conclusion: Plasma HGF and soluble cMet levels were significant predictors of the outcomes of severe AKI patients undergoing CRRT. There was no correlation between plasma HGF and soluble cMet levels and fluid balance.

Keywords: Acute kidney injury, Biomarkers, Continuous renal replacement therapy, Hepatocyte growth factor, Soluble c-Met

Received: December 15, 2020; Revised: May 26, 2021; Accepted: June 7, 2021

Editor: Eun Hui Bae, Chonnam National University, Gwangju, Republic of Korea

Correspondence: Jung Pyo Lee

Department of Internal Medicine, Seoul National University Boramae Medical Center, Seoul National University College of Medicine, 20 Boramaero 5-gil, Dongjak-gu, Seoul 07061, Republic of Korea. E-mail: nephrolee@gmail.com

ORCID: https://orcid.org/0000-0002-4714-126

Copyright (C) 2021 by The Korean Society of Nephrology

(a) This is an Open Access article distributed under the terms of the Creative Commons Attribution Non-Commercial and No Derivatives License (http:// creativecommons.org/licenses/by-nc-nd/4.0/) which permits unrestricted non-commercial use, distribution of the material without any modifications, and reproduction in any medium, provided the original works properly cited. 


\section{Introduction}

Acute kidney injury (AKI) occurs in $10 \%$ of hospitalized patients and $30 \%$ of critically ill patients each year, and its incidence is increasing [1]. Severe AKI is one of the leading causes of death in critically ill patients. Despite recent technical advances in AKI management, mortality rates associated with severe AKI are approximately $40 \%$ to $50 \%[2,3]$.

Reliable biomarkers are important for treating AKI patients and predicting outcomes. Several studies have reported various novel biomarkers for AKI that can detect kidney injury before serum creatinine ( $\mathrm{sCr}$ ), such as tissue inhibitor of metalloproteinase (TIMP)-2, insulin-like growth factor-binding protein 7 (IGFBP7) [4], and urinary matrix metalloproteinase 7 [5]. However, few studies have investigated prognostic predictors of the outcomes of patients with severe AKI undergoing continuous renal replacement therapy (CRRT).

Hepatocyte growth factor (HGF) and its tyrosine kinase receptor, cMet, have important roles in regulating wound healing, cell proliferation, and tissue fibrosis [6-8]. Several experimental studies have indicated that HGF has multiple protective effects on physiological and pathophysiological processes in the kidney, including accelerated DNA synthesis and improved kidney cell regeneration, apoptosis, and necrosis (including that of proximal tubular cells, mesangial cells, podocytes, and endothelial cells) [7,8]. Activation of the HGF/cMet axis can improve acute and chronic kidney disease (CKD) by inhibiting oxidative stress, apoptosis, fibrosis, and inflammation $[9,10]$. We have also confirmed that cMet activation by agonistic antibodies can improve renal fibrosis [11].

A recent study showed that plasma HGF level predicted all-cause mortality and cardiovascular mortality in the general population [12]. Additionally, HGF and cMet can predict clinical outcomes of patients with various cancers $[13,14]$. Recent studies have reported that HGF and cMet are biomarkers for CKD $[15,16]$. However, the clinical predictive abilities of HGF and soluble cMet in AKI patients have not been well studied.

In this study, we investigated whether plasma HGF and soluble cMet levels could predict clinical outcomes of AKI patients who participated in the VENUS (volume management under body composition monitoring in critically ill patients on CRRT) trial. Furthermore, we investigated whether plasma HGF and soluble cMet levels were associated with fluid balance.

\section{Methods}

\section{Study design and population}

A total of 136 severe AKI patients admitted to seven hospitals in Korea who participated in the VENUS trial between 2017 and 2019 were enrolled in this study [3]. The VENUS trial was designed to determine whether bioelectrical impedance analysis-guided fluid management could help achieve euvolemic status in patients treated with CRRT more efficiently than fluid management guided by a generally used quantification method. All participants were selected from patients who were scheduled to undergo CRRT for at least 72 hours. There was no mandatory standardized CRRT protocol. CRRT initiation was decided by each institution's physicians. CRRT settings, effluent dose, and target I/O balance were freely applied to maintain stable blood pressure to meet metabolic demands. In general, blood flow was set to $100-130 \mathrm{~mL} / \mathrm{min}$, and target effluent dose was set to $35 \mathrm{~mL} / \mathrm{kg} / \mathrm{hr}$.

Exclusion criteria were the following: age younger than 18 years; imminent death ( $<24$ hours); maintenance dialysis used before current hospitalization; any other major illness that, according to the investigators' judgment, would substantially increase the risk associated with the subject's participation in this study; and withdrawal of patient consent.

\section{Clinical data and sample collection}

Clinical baseline characteristics data at the time of study enrollment, including age, sex, mean arterial pressure (MAP), body mass index (BMI), laboratory findings, contributing factors for AKI, all-cause mortality, outcome events include sepsis, cardiogenic diseases, pulmonary diseases, and cancer, among others, and Acute Physiology and Chronic Health Evaluation II (APACHE II) scores, and age-adjusted Charlson comorbidity index (Age-CCI) scores, were collected. Age-CCI is the combination of the age equivalence index and Charlson comorbidity index (CCI) [17]. For patients over 40 years old, the cumulative score was 1 point for each additional 10 years of age, and the score for age was added to the CCI. A completely resolved condition or current inactive surgery history was not considered a comorbid disease [18]. 
Laboratory evaluations included measurements of complete blood cell counts, electrolytes, sCr, total protein, albumin, calcium, and high-sensitivity C-reactive protein. Blood samples were centrifuged to extract plasma. The plasma was frozen, stored at $-70^{\circ} \mathrm{C}$, and thawed before analysis.

All procedures were performed in accordance with the ethical standards of the institutional and/or national research committee and the 1964 Declaration of Helsinki and its later amendments or comparable ethical standards. All patients provided written informed consent at the time of their enrollment. The APACHE II score was designed to measure the disease severity of adult patients admitted to the intensive care unit (ICU) during the first 24 hours after admission. It is calculated using the patient's age and 12 routine physiological measurements (alveolar-arterial oxygen gradient $\left[\mathrm{AaDO}_{2}\right]$ or partial pressure of oxygen $\left[\mathrm{PaO}_{2}\right]$ depending on the fraction of inspired oxygen $\left[\mathrm{FiO}_{2}\right]$, temperature, MAP, $\mathrm{pH}$, heart rate, respiratory rate, sodium level, potassium level, creatinine level, hematocrit, white blood cell count, and Glasgow Coma Scale score). Approval to perform the study was obtained from the Institutional Review Boards of all participating centers (No. 20-2019-79). The trial protocol has been registered at http://www.clinicaltrials.gov (NCT03330626).

\section{Measurement of plasma soluble cMet and hepatocyte growth factor}

Enzyme-linked immunosorbent assay kits were used to measure the plasma concentrations of HGF (DY294; Becton Dickinson, Minneapolis, MN, USA) and soluble cMet (KHO 2031; Invitrogen, Vienna, Austria) on day (D) 0, D2, and D7. Plasma HGF concentrations were measured after a 10-fold dilution and plasma soluble cMet concentration was measured after a two-fold dilution. Enzyme-linked immunosorbent assays was performed according to the manufacturers' instructions. All laboratory investigators were blinded to the sample source, and all measurements were performed in duplicate.

\section{Assessment of fluid status}

Fluid status was measured by bioimpedance analysis using InBody S10 (InBody, Seoul, Korea) on D0, D2, and D7. Measurements of total body water (TBW), intracellular water
(ICW), and extracellular water (ECW) were obtained with InBody S10. Fluid state was based on ECW/TBW and TBW/ height squared $\left(\mathrm{H}^{2}\right)$.

\section{Clinical outcomes}

Primary outcome was all-cause mortality after CRRT. We investigated the associations between HGF concentration and soluble cMet concentration and all-cause mortality. In this study, survival duration was calculated from the day of enrollment in the study to the day of discharge from the hospital or death. The relationship between all-cause mortality after 2 years of follow-up was also assessed, starting from the first enrolled patient. Secondary outcome was fluid status according to HGF and soluble cMet concentrations.

\section{Statistical analysis}

Patients were classified into three groups based on their D0 HGF and soluble cMet concentrations. Table 1 shows the HGF and soluble cMet concentrations of each group. Categorical variables, which were expressed as frequencies and proportions, were compared using the chi-square tests. After testing for normality, normally distributed continuous variables were expressed as mean \pm standard deviations and compared using Student t test or one-way analysis of variance. Nonnormally distributed variables were expressed as medians (interquartile ranges) and compared using the Mann-Whitney U or Kruskal-Wallis tests. To investigate the impact of D0, D2, and D7 plasma soluble cMet and HGF levels on mortality, Kaplan-Meier survival curves were constructed. Cox proportional hazard models with plasma soluble cMet or HGF levels were used for multivariate survival analyses. To examine the prognostic value of plasma HGF and soluble cMet levels at multiple time points, we calculated the statistical significance of differences between the areas under the curve (AUC) at three-time points (D0, D2, and D7). Survival duration was calculated from day 0 to evaluate the predictive effects of D2 and D7 soluble cMet and HGF. Receiver operating characteristic (ROC) curves and AUC were generated to evaluate the accuracy and predictive capability of each indicator of survival. To examine the incremental prognostic value before and after inclusion of plasma HGF and soluble cMet levels with traditional indices, including APACHE II and Age-CCI, we calculated the statis- 


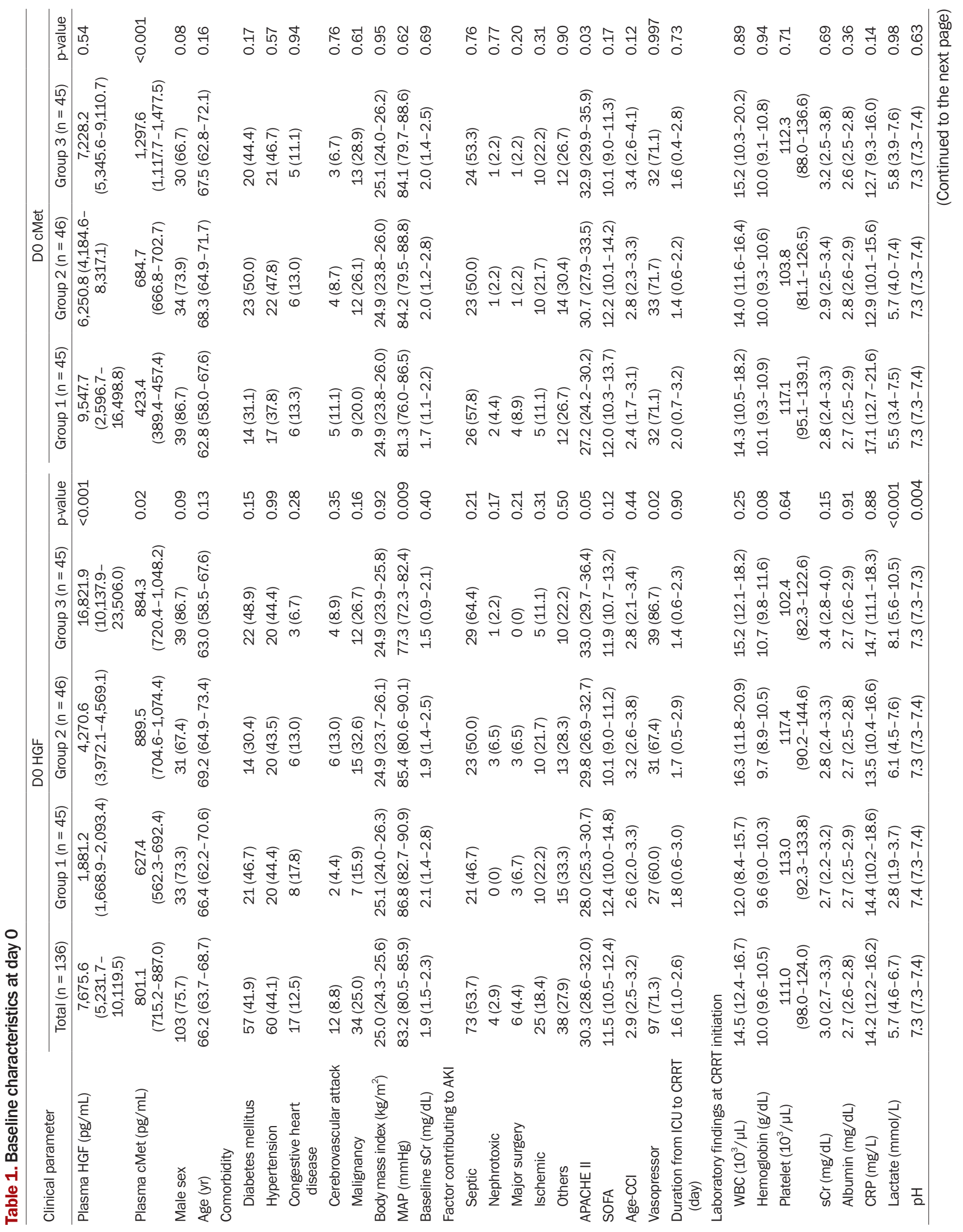




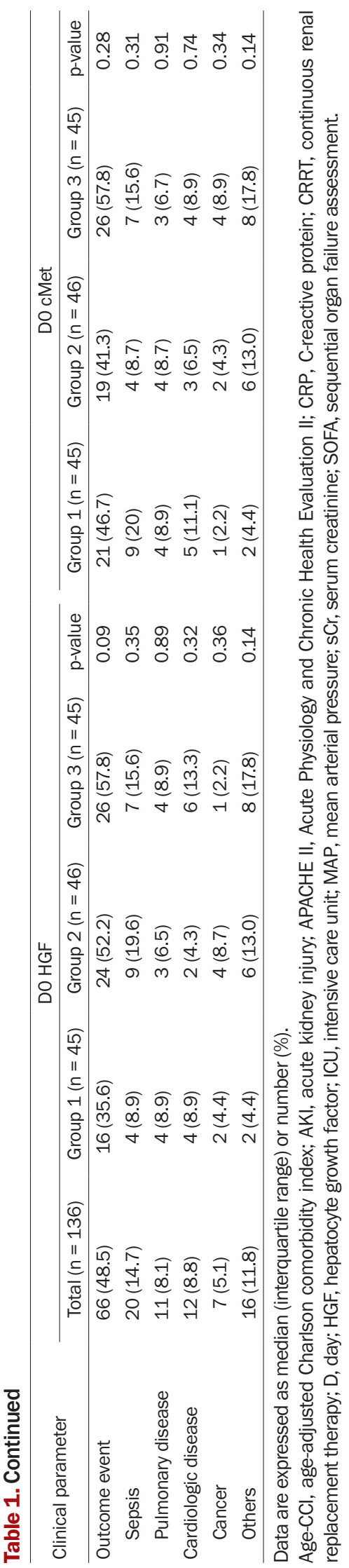

tical significance of differences between the AUC, integrated discrimination improvement (IDI) index, and category-free net reclassification improvement (cfNRI) index. We divided patients into four groups based on median HGF and soluble cMet levels to determine the relationship between the two indicators and mortality rate. Patients with low concentrations of HGF and soluble cMet were classified into group 1; individuals with high concentrations of both HGF and soluble cMet were classified into group 4 . Those patients with a low HGF concentration but high soluble cMet concentration were assigned to group 2; those with a high concentration of HGF combined with a low concentration of soluble cMet were assigned to group 3 . We evaluated fluid status on D0, D2, and D7 according to HGF and soluble cMet concentrations using Student t test. All statistical analyses were performed using IBM SPSS version 23 (IBM Corp., Armonk, NY, USA) and R program version 3.2.5 (The R Foundation for Statistical Computing, Vienna, Austria). The p-value of $<0.05$ indicated statistical significance.

\section{Results}

\section{Baseline characteristics}

The study cohort consisted of 136 patients divided into three groups based on their HGF and soluble cMet concentrations on D0. Table 1 provides clinical baseline characteristics based on D0 HGF and soluble cMet concentrations. There were no significant differences in diabetes, hypertension, $\mathrm{BMI}, \mathrm{sCr}$, or blood urea nitrogen among the three groups based on D0 HGF and soluble cMet concentrations. In the group with the highest D0 HGF concentration, the soluble cMet concentration was significantly higher $(\mathrm{p}=0.02)$, MAP was significantly lower ( $\mathrm{p}=0.009)$, and more patients were on vasopressors ( $\mathrm{p}$ $=0.02$ ). The laboratory findings of patients in the highest $\mathrm{D} 0$ HGF group indicated higher lactate levels in this group $(\mathrm{p}<$ $0.001)$ and a lower $\mathrm{pH}(\mathrm{p}=0.004)$. The group with the highest D0 soluble cMet concentration had a higher APACHE II score than the other two groups $(\mathrm{p}=0.03)$.

\section{Variations and correlations between hepatocyte growth factor and soluble cMet concentrations after continuous renal replacement therapy}

Both HGF and soluble cMet concentrations tended to 
decrease after CRRT. HGF concentrations on D7 were significantly reduced compared to those on D0 (Fig. 1A). Concentrations of HGF and soluble cMet on D0 were positively correlated with each other $\left(R^{2}=0.126 ; p=0.03\right.$ ) (Fig. $1 B$ ). HGF and soluble cMet concentrations were not significantly correlated on $\mathrm{D} 2\left(\mathrm{R}^{2}=0.107\right.$ and $\left.\mathrm{p}=0.09\right)$ or $\mathrm{D} 7\left(\mathrm{R}^{2}=0.06\right.$ and $\mathrm{p}=0.42)($ Fig. 1C, D).

Effects of plasma hepatocyte growth factor levels on clinical outcomes

Kaplan-Meier survival curves and log-rank tests were used to investigate the associations between plasma HGF concentrations on D0, D2, and D7 and mortality. We divided patients into three groups and analyzed survival rates. Survival rate distribution of the first and second groups based on HGF and soluble cMet levels were similar, but different from those of the third group that had the highest concentration of both HGF and soluble cMet. Therefore, we combined the first group and the second group for analysis (Supplementary Fig. 1, available online). The risk of all-cause mortality was increased in patients with the highest D0 HGF levels $(\mathrm{p}=0.04)$ (Fig. 2A). D2 and D7 HGF levels displayed similar associations with in-hospital mortality, especially D7 HGF level ( $\mathrm{p}=0.01$ and $\mathrm{p}=0.001$, respectively) (Fig. 2B, C). In the 2-year follow-up data, plasma D0, D2, and D7 HGF levels were still closely related to all-cause mortality (Supplementary Fig. 2A-C, available online). Next, we performed multivariate Cox proportional analysis to investigate the independent effects of D0, D2, and D7 HGF levels on patient outcomes (Table 2). Elevated HGF values remained an independent variable associated with clinical outcomes after adjusting for confounding variables, including sex, BMI, APACHE II score, and Age-CCI (D0 HGF: hazard ratio [HR] $1.71,95 \%$ confidence intervals [CI] 1.02-2.86, $\mathrm{p}=0.04$; D2 HGF: HR 2.57, 95\% CI 1.37-4.83, $\mathrm{p}=0.003$; D7 HGF: HR 4.26,
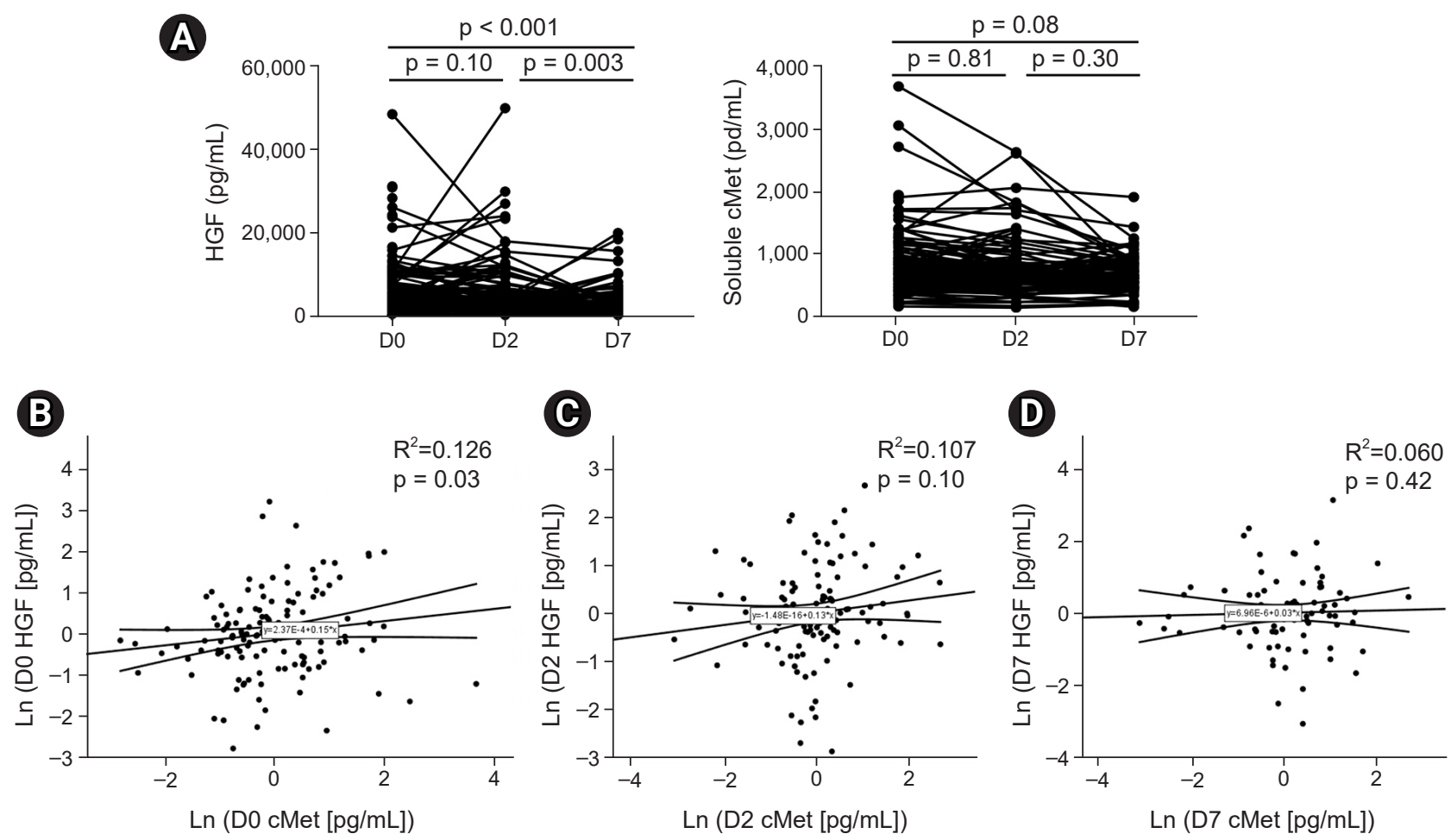

Figure 1. Variations and correlations between HGF and soluble cMet levels. (A) Trends of HGF and soluble cMet concentrations in plasma after CRRT. (B) Plasma HGF level converted to a natural logarithm was positively correlated with plasma soluble cMet level on DO after CRRT was initiated. Pearson correlation coefficient was $\mathrm{R}^{2}=0.126$ with a $\mathrm{p}$-value of 0.03 . No correlation was observed between plasma HGF levels and cMet levels on D2 (C) or D7 (D) after initiating CRRT.

CRRT, continuous renal replacement therapy; D, day; HGF, hepatocyte growth factor. 

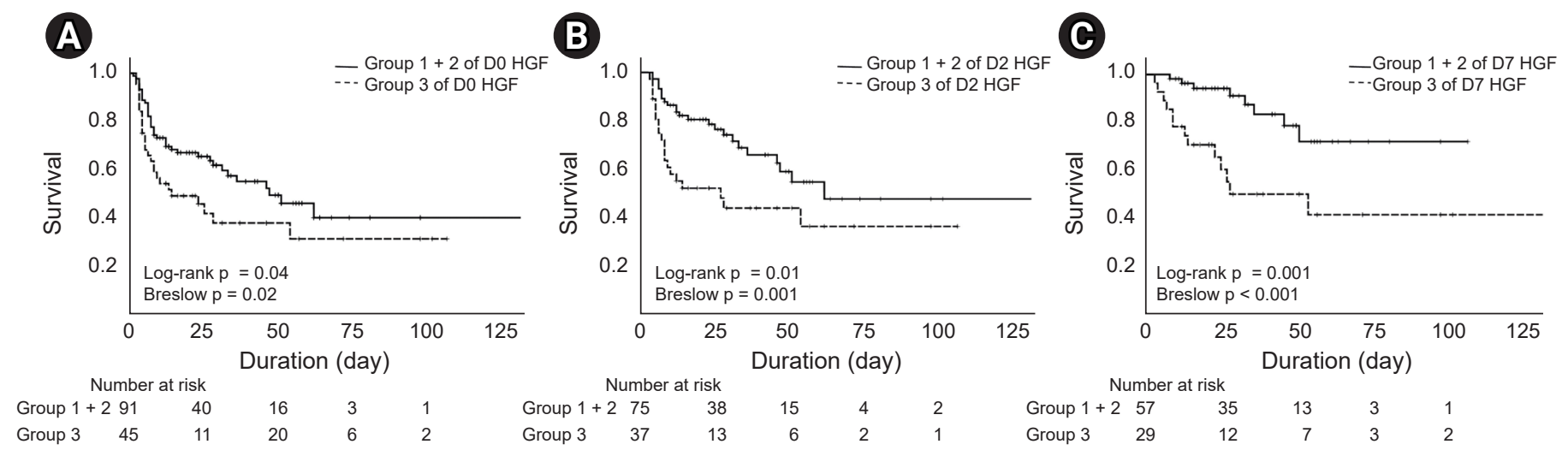

\begin{tabular}{lrrrrr}
\multicolumn{7}{c}{ Number at risk } \\
Group 1 2 & 57 & 35 & 13 & 3 & 1 \\
Group 3 & 29 & 12 & 7 & 3 & 2
\end{tabular}
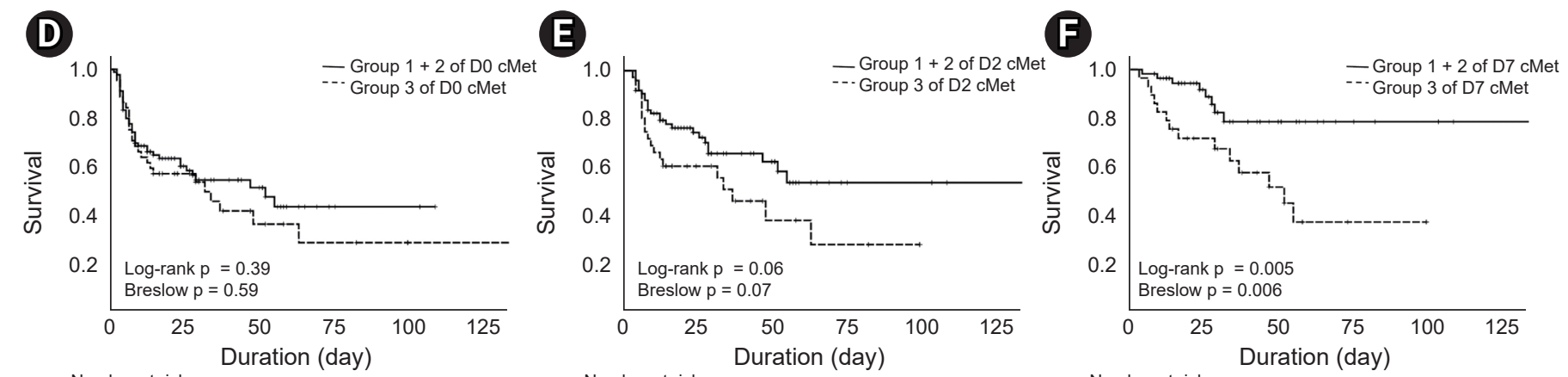

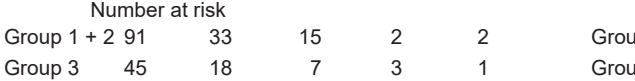

\begin{tabular}{lrrrrrr}
\multicolumn{7}{c}{ Number at risk } \\
Group 1 +2 & 75 & 36 & 16 & 3 & 3 & Group 1 \\
Group 3 & 37 & 14 & 5 & 3 & 0 & Group
\end{tabular}

\begin{tabular}{lrrrrr}
\multicolumn{6}{c}{ Number at risk } \\
Group 1 2 & 57 & 31 & 12 & 4 & 3 \\
Group 3 & 29 & 17 & 8 & 2 & 0
\end{tabular}

Figure 2. Survival rates of AKI patients who underwent CRRT according to plasma HGF and soluble cMet concentrations. (A) Patients in group 3 had a significantly lower survival rate than patients in group 1 and group 2 according to plasma HGF concentration on DO (log-rank $p=0.04$ ). (B and $C$ ) Patients in HGF group 3 had a significantly lower survival rate than those in groups 1 and 2 on D2 (log-rank $p=0.01$ ) and D7 (log-rank $p=0.001$ ). Patients in group 3 were divided according to plasma soluble cMet concentrations on D0, D2, and D7. (D and E) No difference in mortality rates was found between cMet groups 1 and 2 and group 3 on D0 and D2. (F) The risk of all-cause mortality was significantly increased in the patient group with the highest D7 cMet levels $(p=0.005)$.

AKI, acute kidney injury; CRRT, continuous renal replacement therapy; D, day; HGF, hepatocyte growth factor.

95\% CI 1.71-10.62, $\mathrm{p}=0.002$ ).

Additionally, we evaluated the effects of changes in HGF concentrations between different time points during the week after CRRT initiation on clinical outcomes. Changes in HGF concentrations were calculated as differences between D2 and D0, between D7 and D0, and between D7 and D2. Changes in HGF concentrations were not related to mortality (Supplementary Fig. 3A-C, available online).

\section{Association between plasma soluble cMet level and} clinical outcomes

We used the same method to investigate the associations between plasma soluble cMet concentrations on D0, D2, and D7 and in-hospital mortality. D0 and D2 soluble cMet levels did not predict all-cause mortality (Fig. 2D-E). On D7, the risk of all-cause mortality (Fig. 2F) was significantly increased in the patient group with the highest soluble cMet level ( $p=0.005)$. In the 2-year follow-up data, plasma D0 soluble cMet level was still closely related to all-cause mortality (Supplementary Fig. 2D-F). Multivariate Cox regression analysis showed that the group with the highest soluble cMet level had a significantly higher mortality rate than the other groups after adjusting for other risk factors such as sex, BMI, APACHE II score, and Age-CCI (HR, 4.18; 95\% CI, 1.69-10.32; $\mathrm{p}=0.002$ ) (Table 3). Additionally, changes in soluble cMet concentrations between D2 and D0, between D7 and D0, and between D7 and D2 were not associated with all-cause mortality (Supplementary Fig. 3D-F). 

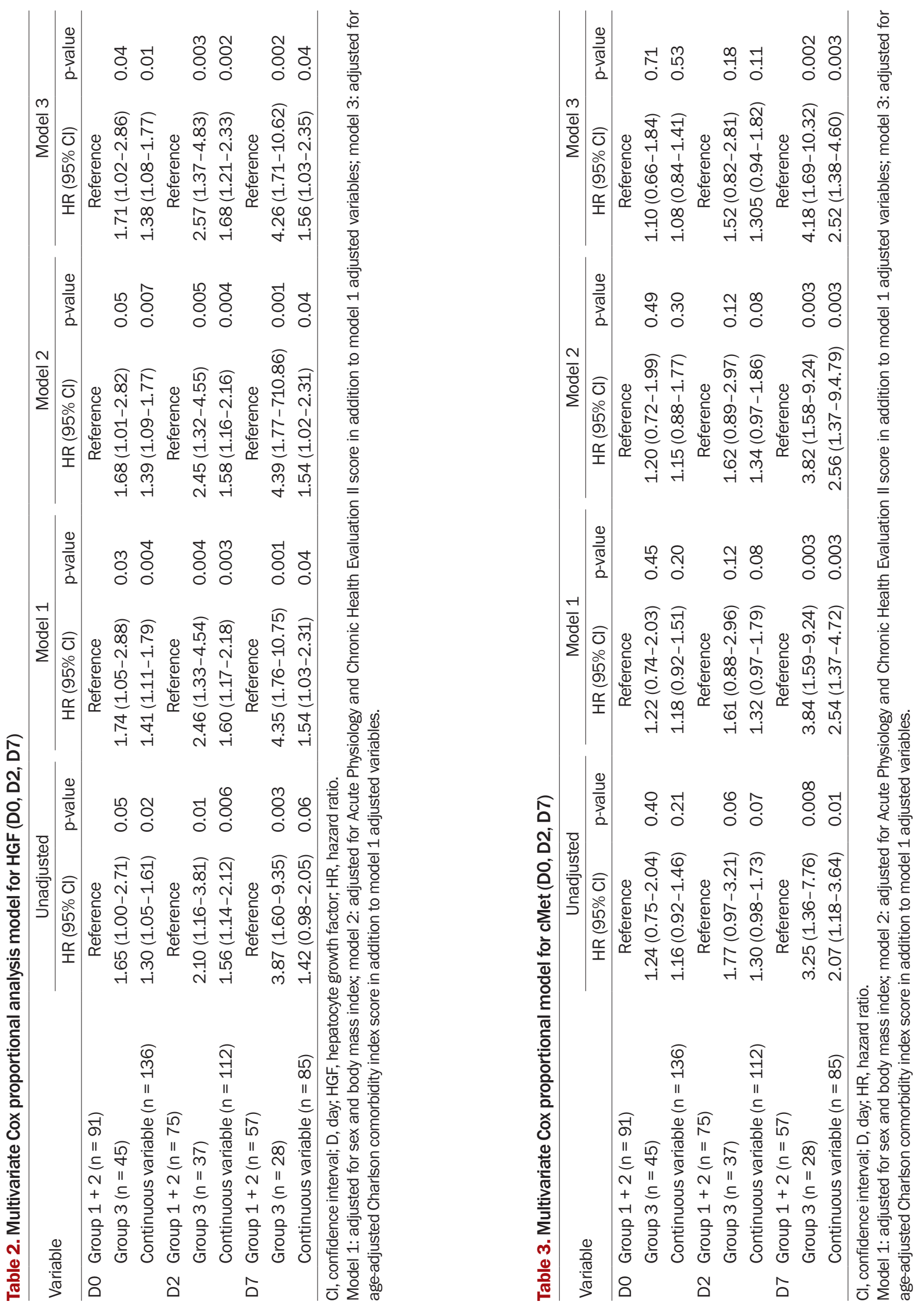
Ability of plasma hepatocyte growth factor and soluble cMet to predict outcomes at different time points

Levels of HGF and soluble cMet that best predicted mortality were investigated using ROC curves. The AUCs (95\% CI) of D0, D2, and D7 HGF were 0.576 (0.440-0.711), 0.540 (0.3920.689 ), and 0.716 (0598-0.838), respectively (Fig. 3A). The AUCs (95\% CI) of D0, D2, and D7 soluble cMet were 0.579 (0.440-0.719), 0.570 (0.429-0.711), and 0.678 (0.553-0.825), respectively (Fig. 3B).

Plasma hepatocyte growth factor and soluble cMet as biomarkers for predicting mortality

Next, we evaluated whether the addition of plasma HGF and soluble cMet to conventional prognostic markers such as APACHE II and Age-CCI improved prediction of mortality (Table 4). In ROC analysis, the addition of $\log$ (D7 HGF) and $\log$ (D7 cMet) increased the AUC. The AUCs (95\% CIs) of the APACHE II (model 1), APACHE II + $\log$ (D7 HGF) (model 2), and APACHE II + Age-CCI + $\log ($ D7 HGF $)+\log (\mathrm{D} 7 \mathrm{cMet})$ (model 3) were 0.523 (0.393-0.0.653), 0.710 (0.588-0.832), and 0.749 (0.635-0.863), respectively. Additionally, the
IDI and cfNRI for predicting mortality (model 1 vs. model 3) were $0.166(0.070-0.262 ; \mathrm{p}<0.001)$ and $75.1 \%(29.9 \%-$ $120.3 \%$; $<<0.001$ ), suggesting that addition of plasma $\log (\mathrm{D} 7$ HGF) and $\log (\mathrm{D} 7 \mathrm{cMet})$ to conventional predictors of mortality significantly increased predictive value.

Prediction of clinical outcomes according to combined hepatocyte growth factor and soluble cMet concentrations

The ability to predict patient outcomes using the combination of HGF and soluble cMet concentrations was evaluated. Group 1 had a significantly higher mortality rate than the other groups $(p=0.04)$. Furthermore, the difference in mortality between these groups was statistically significant when using D7 HGF and cMet concentrations ( $\mathrm{p}=0.02$ ) (Supplementary Fig. 4A-C, available online).

Fluid status according to hepatocyte growth factor and soluble cMet concentrations

We investigated fluid status according to HGF and soluble cMet concentrations using parameters of fluid status measured using InBody S10. There was no significant difference
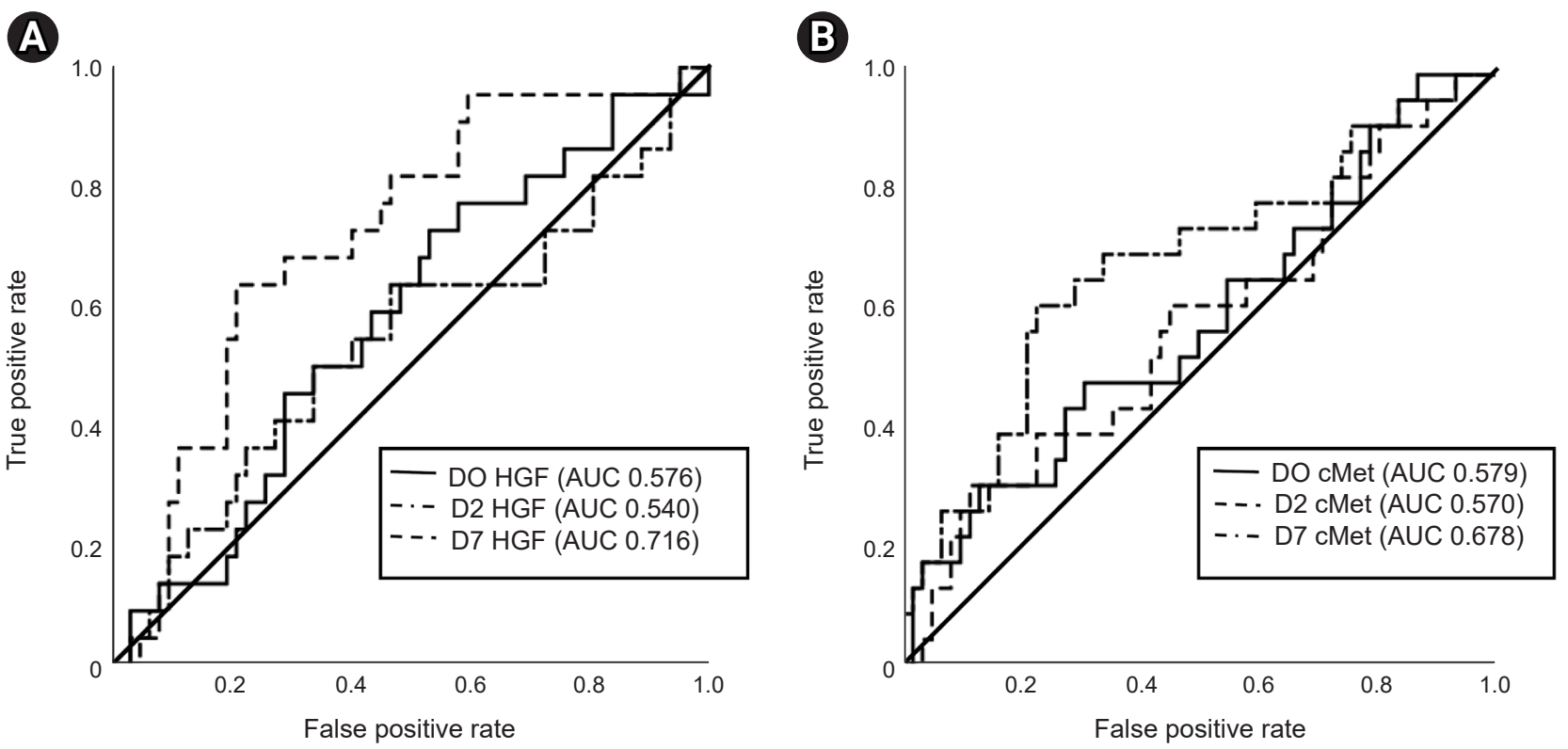

Figure 3. Comparison of the ROC curves for plasma HGF and soluble cMet at various time points. (A) ROC curve and AUC for plasma HGF concentrations at various time points. (B) ROC curve and AUC for plasma soluble cMet concentrations at various time points.

AUC, area under the curve; D, day; HGF, hepatocyte growth factor; ROC, receiver operating characteristic. 


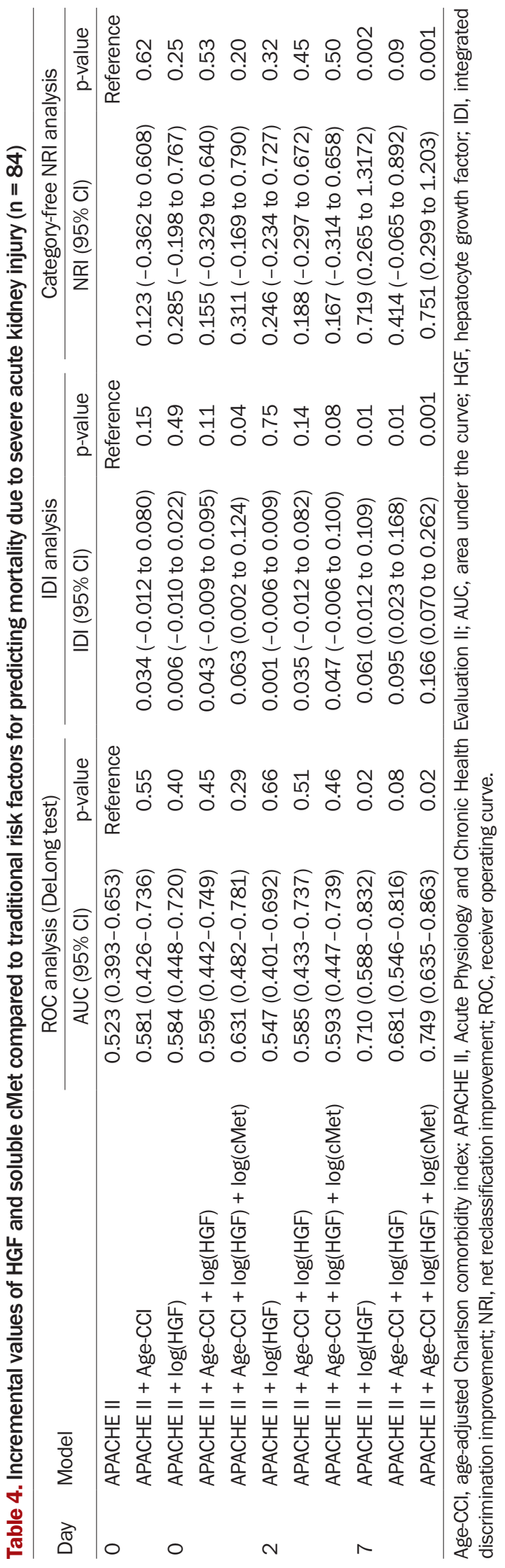

between ECW/TBW and plasma HGF and soluble cMet levels on D0, D2, or D7 (Fig. 4A). There was no significant difference between plasma HGF levels on D0 and D7 and soluble cMet levels on D0, D2, and D7. TBW $/ \mathrm{H}^{2}$ increased significantly $(p=0.02)$ only in the group of patients with the highest D2 HGF level (Fig. 4B).

\section{Discussion}

In this study, we found that increased plasma HGF and soluble cMet levels could predict the outcomes of patients with severe AKI undergoing CRRT. Plasma HGF and soluble cMet levels on D7 were the most valuable predictors of patient outcomes. The inclusion of plasma HGF and soluble cMet levels with conventional prognostic index markers improved the predictive power of the composite indices. Finally, there was no correlation between plasma HGF and soluble cMet levels and fluid balance. To our knowledge, this is the first study to show that increased plasma HGF and soluble cMet levels of patients with severe AKI can predict clinical outcomes.

HGF and its receptor cMet are key components of a signaling pathway with critical roles in cellular regeneration, proliferation, differentiation, invasion, angiogenesis, antiapoptosis, tissue fibrosis, and wound healing $[19,20]$. The HGF/ cMet signaling pathway has therefore been identified as a target to alleviate the various types of AKI and prevent progression to CKD. The expression of HGF has been shown to be increased in various acute and CKDs including experimental acute ischemic injury, toxic elements, and unilateral nephrectomy [21,22].

HGF may act as an endocrine and paracrine effector for kidney repair during kidney injury. Administration of exogenous HGF promotes tubular repair and recovery [22]. Previously, we demonstrated that administration of cMet agonistic antibodies halted the progression of CKD [11]. Our group also confirmed that cMet agonistic antibodies attenuate apoptosis in AKI [23]. Conversely, disruption of HGF signaling aggravates renal interstitial fibrosis after obstructive injury [24]. However, many studies have reported that elevated plasma HGF and soluble cMet concentrations are associated with poorer clinical prognosis. Santalahti et al. [12] reported that levels of HGF and placental growth factor in plasma could predict mortality in a general population. Elevated circulating HGF levels have been observed in many patho- 
(4)
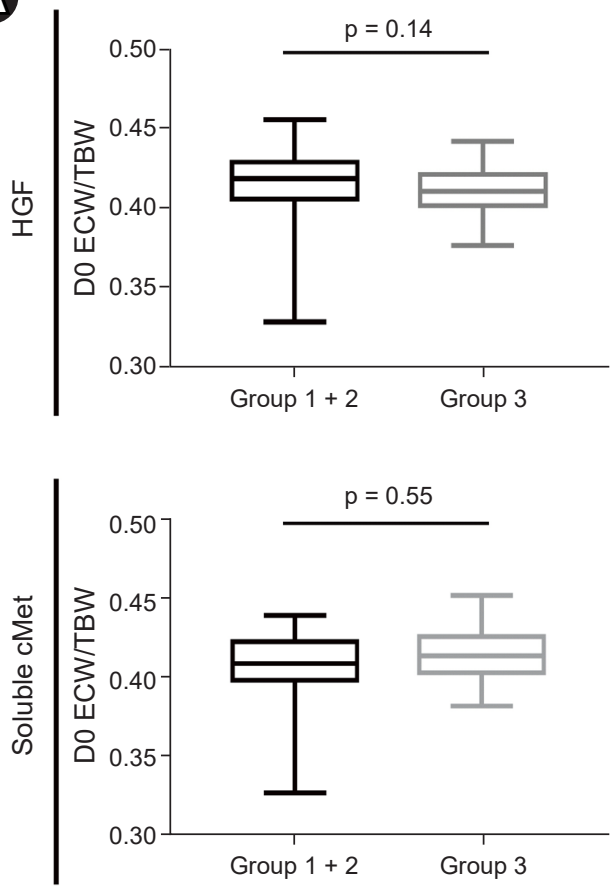

B
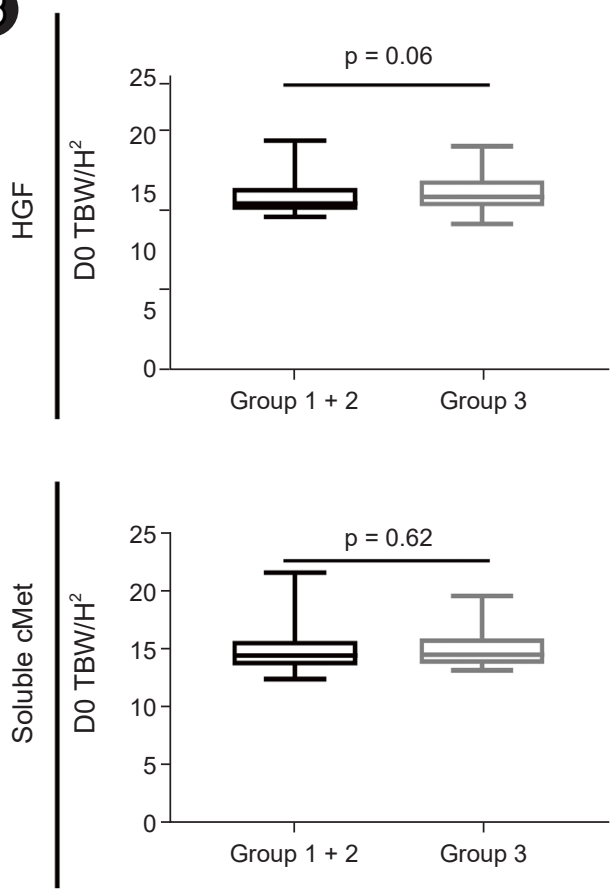
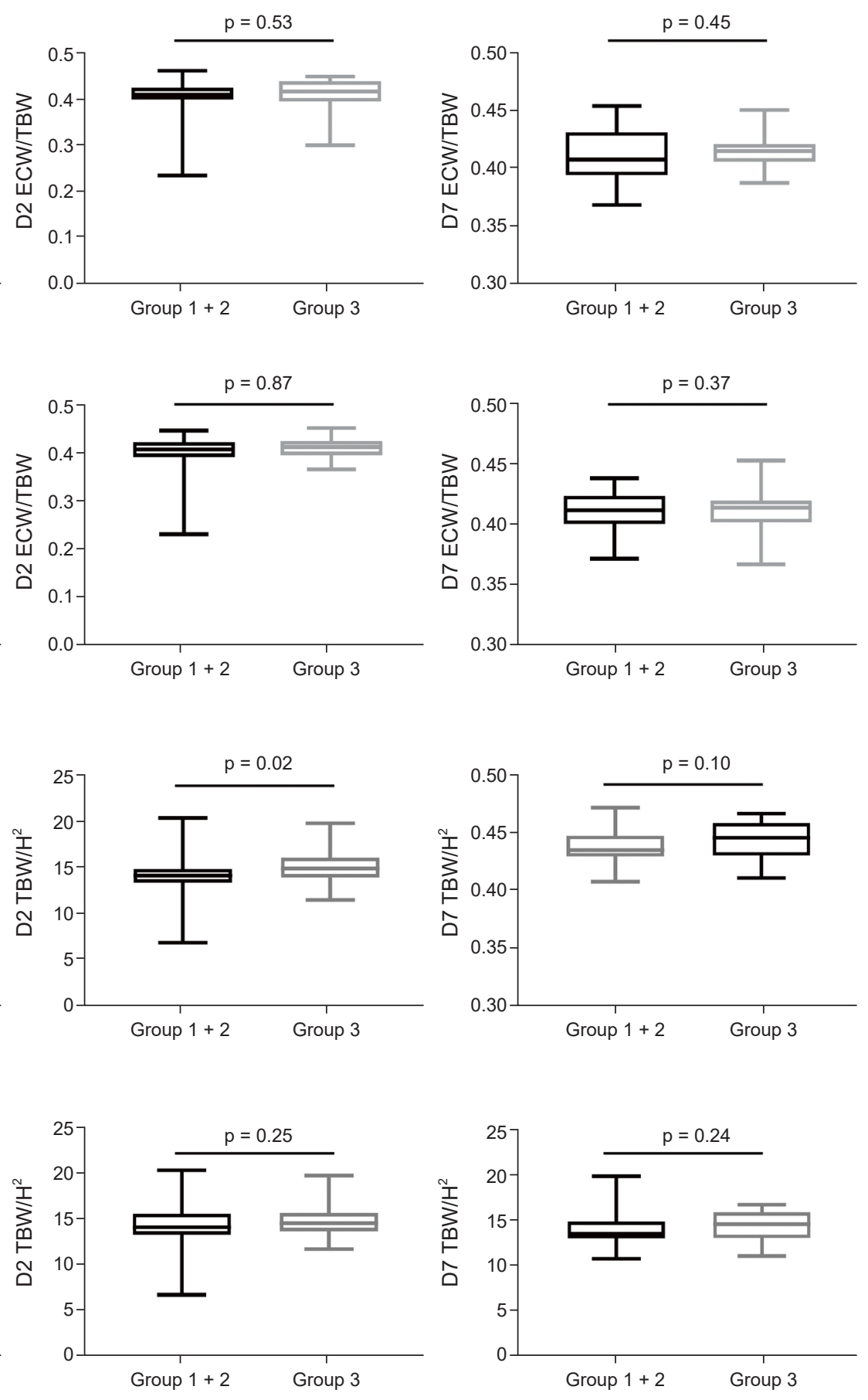

Figure 4. Euvolemic status on D7 after CRRT according to HGF and soluble cMet concentrations. (A) Comparison of ECW/TBW and plasma HGF or soluble cMet on DO, D2, and D7. (B) Comparison of TBW/ $\mathrm{H}^{2}$ and plasma HGF or soluble cMet levels.

CRRT, continuous renal replacement therapy; D, day; ECW, extracellular water; HGF, hepatocyte growth factor; $\mathrm{H}^{2}$, height squared; TBW, total body water. 
logic liver diseases, including hepatitis and hepatocellular carcinoma, and have been shown to be correlated with more severe liver cirrhosis [25]. Likewise, HGF levels are associated with sepsis and correlated with established markers of endothelial cell injury. Elevated HGF level in sepsis patients is a significant indicator of a poor prognosis [26]. Moreover, an increase in soluble cMet concentration has been shown to be associated with a poor clinical prognosis. As reported in various malignant tumors, soluble cMet concentration is associated with progression, metastasis, and a poor prognosis [27-29]. Our group previously demonstrated increased urinary cMet in diabetic nephropathy patients was strongly associated with progression to end-stage renal disease [15].

There are several explanations for why elevated HGF and soluble cMet concentrations are highly related to a poorer prognosis. First, the greater the extent of the disease, the greater the activation of the HGF/cMet signaling pathway for cell regeneration. Overexpression of the HGF/cMet pathway and increased plasma HGF and soluble cMet concentrations may be closely related to disease progression. In addition, an increase in soluble cMet inhibits the phosphorylation of cMet, thereby inhibiting the HGF/cMet signaling pathway [30].

One study reported a marked increase in urinary HGF levels in patients with AKI [23]. Another study showed that HGF concentration in serum was significantly increased in AKI patients relative to participants receiving hemodialysis (approximately 20-fold) [31]. Clinical trials have indicated that HGF and other biomarkers in urine (such as neutrophil gelatinase-associated lipocalin [NGAL]) have the potential to predict AKI [32]. These studies suggested that HGF was correlated with AKI severity. Despite these data, these studies only examined AKI patients with urine volumes that were maintained and measured at one-time point; furthermore, cMet concentration was not considered. Additionally, the mortality rate of patients was not assessed.

We previously reported that the expression of HGF and soluble cMet in plasma was significantly increased in AKI patients [23]. In this study, we evaluated if plasma HGF and soluble cMet concentrations could predict clinical longitudinal outcomes of severe AKI patients undergoing CRRT. We confirmed that mortality rates during hospitalization were significantly higher in patients with severe AKI and patients with the highest HGF levels on D0, D2, and D7. Soluble cMet level on D7 was a significant predictor of patient mortality.
After adjusting for sex, BMI, APACHE II score, Age-CCI, HGF and soluble cMet levels were still significant predictors of patient mortality. A large amount of HGF may be released as an injury protection mechanism to activate downstream signaling pathways. HGF is released not only by damaged kidneys but also distant organs (lung, liver, spleen) and can participate in tubular repair both as an endocrine factor and paracrine substance [33]. This suggests that plasma HGF and soluble cMet levels can be used as predictors of the prognosis of patients with severe AKI and of clinical risk and recovery after AKI.

A previous study showed that a panel of urine biomarkers measured on D1, D7, and D14 yielded significantly different results for those recovering from AKI compared with those patients who did not recover [34]. We analyzed the correlation between plasma HGF and soluble cMet concentrations and mortality at multiple time points after the initiation of CRRT (D0, D2, and D7). We found that plasma HGF as a clinical predictor was not affected by CRRT and that plasma HGF was a better predictor of mortality than soluble cMet. Furthermore, we examined whether HGF and soluble cMet levels on D0, D2, and D7 were reliable biomarkers of mortality using ROC curves and AUCs. D7 HGF and soluble cMet concentrations were prognostic biomarkers with high sensitivity and specificity. The best time to measure plasma HGF and soluble cMet levels may be on D7 after the start of CRRT for severe AKI; repeated measurements can improve prediction accuracy.

An individual biomarker is rarely sufficient for clearly defining a particular pathologic state [35,36]. Vaidya et al. [32] measured kidney injury molecule-1, HGF, NGAL, and interleukin-18 levels simultaneously in the same aliquot of urine. The specificity and sensitivity of the combination of these urinary biomarkers for the diagnosis of AKI were significantly higher than those of single biomarkers. The urinary [TIMP-2] - [IGFBP7] test can be used to identify critically ill patients at high risk for imminent AKI [5]. Therefore, multiple biomarkers measured in the same biological sample at the same time are extremely useful for predicting outcomes. Circulating HGF and cMet are attractive potential alternative biomarkers for ligand overexpression and receptor overexpression, respectively [37]. In this study, we analyzed the ability of combined plasma HGF and soluble cMet levels to predict mortality in patients with severe AKI. We found that the mortality rates of groups with high HGF and soluble 
cMet concentrations on D2 and D7 were significantly higher than those of other groups; furthermore, the inclusion of soluble cMet and HGF with conventional prognostic indices such as APACHE II and Age-CCI significantly improved predictive power.

Rhee et al. [38] demonstrated that TBW/ $\mathrm{H}^{2}\left(\geq 13 \mathrm{~L} / \mathrm{m}^{2}\right)$ and ICW/ $\mathrm{H}^{2}$ were independently associated with higher in-hospital mortality for patients with AKI undergoing CRRT. That study showed that the fluid status could be assessed using ECW/TBW in critically ill patients requiring CRRT and that ECW/TBW could predict mortality [39]. All patients who participated in this study were admitted to the ICU, and their fluid balance was maintained during CRRT. We used ECW/ TBW to evaluate the correlation between fluid status and plasma HGF or soluble cMet levels; however, no significant correlation was found.

The present study had some limitations. First, although ethnicity can influence several clinical outcomes, data were acquired only from a Korean population, and this study was limited to Korean tertiary hospitals. Second, only mortality during hospitalization was assessed and long-term outcomes were not considered. Finally, plasma HGF and soluble cMet concentrations could be affected by CRRT. It is necessary to identify HGF and soluble cMet levels in CRRT waste liquid or blood before and after CRRT to confirm that the CRRT waste liquid does not contain HGF or soluble cMet in future studies.

In summary, plasma HGF measurements at multiple time points predicted the clinical outcomes of patients with severe AKI undergoing CRRT. Furthermore, plasma soluble cMet measurements at a specific time point were able to predict clinical outcomes. For accurate mortality predictions, the most valuable time to perform plasma HGF and soluble cMet measurements is D7 after CRRT, and repeated measurements can improve accuracy. We found no correlation between plasma HGF and soluble cMet levels and fluid balance in this study.

\section{Conflicts of interest}

All authors have no conflicts of interest to declare.

\section{Funding}

This work was supported by a grant from the Korea Health
Technology R\&D Project through the Korea Health Industry Development Institute (KHIDI), funded by the Ministry of Health and Welfare, Republic of Korea (grant number: HI17C1827) in addition to a grant from the Korea Healthcare Technology R\&D Project, Ministry for Health and Welfare, Republic of Korea (HI17C1693).

\section{Acknowledgments}

The authors would like to thank the study participants. The biospecimens and data used for the present study were provided by the Biobank of Seoul National University Hospital, a member of the Korea Biobank Network (KBN4 A03).

\section{Authors' contributions}

Conceptualization: LL, JPL

Funding acquisition: JPL

Investigation, Methodology: LL, JNA, JL, JHK, SMZ, DJS, JPL

Formal analysis, Visualization: LL, JNA

Supervision, Validation: JL, DKK, DRR, SK

Writing-original draft: LL, JPL

Writing-review \& editing: All authors

All authors read and approved the final manuscript.

\section{ORCID}

Lilin Li, https://orcid.org/0000-0001-7559-1018

Jung Nam An, https://orcid.org/0000-0001-5108-1005

Jeonghwan Lee, https://orcid.org/0000-0003-3199-635X

Dong Jin Shin, https://orcid.org/0000-0002-8122-6786

Shi Mao Zhu, https://orcid.org/0000-0002-1020-4969

Jin Hyuk Kim, https://orcid.org/0000-0001-9336-1750

Dong Ki Kim, https://orcid.org/0000-0002-5195-7852

Dong-Ryeol Ryu, https://orcid.org/0000-0002-5309-7606

Sejoong Kim, https://orcid.org/0000-0002-7238-9962

Jung Pyo Lee, https://orcid.org/0000-0002-4714-1260

\section{References}

1. An JN, Hwang JH, Kim DK, et al. Chronic kidney disease after acute kidney injury requiring continuous renal replacement therapy and its impact on long-term outcomes: a multicenter retrospective cohort study in Korea. Crit Care Med 2017;45:4757. 
2. Oh HJ, An JN, Oh S, et al. VolumE maNagement Under body composition monitoring in critically ill patientS on CRRT: study protocol for a randomized controlled trial (VENUS trial). Trials 2018;19:681.

3. Oh HJ, Shin DH, Lee MJ, et al. Early initiation of continuous renal replacement therapy improves patient survival in severe progressive septic acute kidney injury. J Crit Care 2012;27:743.

4. Bihorac A, Chawla LS, Shaw AD, et al. Validation of cell-cycle arrest biomarkers for acute kidney injury using clinical adjudication. Am J Respir Crit Care Med 2014;189:932-939.

5. Yang X, Chen C, Teng S, et al. Urinary matrix metalloproteinase-7 predicts severe AKI and poor outcomes after cardiac surgery. J Am Soc Nephrol 2017;28:3373-3382.

6. Ishibe S, Haydu JE, Togawa A, Marlier A, Cantley LG. Cell confluence regulates hepatocyte growth factor-stimulated cell morphogenesis in a beta-catenin-dependent manner. Mol Cell Biol 2006;26:9232-9243.

7. Gong R, Rifai A, Dworkin LD. Hepatocyte growth factor suppresses acute renal inflammation by inhibition of endothelial E-selectin. Kidney Int 2006;69:1166-1174.

8. Zhang C, Hou B, Yu S, Chen Q, Zhang N, Li H. HGF alleviates high glucose-induced injury in podocytes by GSK3 $\beta$ inhibition and autophagy restoration. Biochim Biophys Acta 2016;1863:2690-2699.

9. Dai C, Saleem MA, Holzman LB, Mathieson P, Liu Y. Hepatocyte growth factor signaling ameliorates podocyte injury and proteinuria. Kidney Int 2010;77:962-973.

10. Gattai PP, Maquigussa E, da Silva Novaes A, et al. miR-26a modulates HGF and STAT3 effects on the kidney repair process in a glycerol-induced AKI model in rats. J Cell Biochem 2018; 119:7757-7766.

11. Kim YC, Lee J, An JN, et al. Renoprotective effects of a novel cMet agonistic antibody on kidney fibrosis. Sci Rep 2019;9:13495.

12. Santalahti K, Havulinna A, Maksimow M, et al. Plasma levels of hepatocyte growth factor and placental growth factor predict mortality in a general population: a prospective cohort study. $J$ Intern Med 2017;282:340-352.

13. Matsumoto K, Umitsu M, De Silva DM, Roy A, Bottaro DP. Hepatocyte growth factor/MET in cancer progression and biomarker discovery. Cancer Sci 2017;108:296-307.

14. Moosavi F, Giovannetti E, Saso L, Firuzi O. HGF/MET pathway aberrations as diagnostic, prognostic, and predictive biomarkers in human cancers. Crit Rev Clin Lab Sci 2019;56:533-566.

15. Kim YC, An JN, Kim JH, et al. Soluble cMet levels in urine are a significant prognostic biomarker for diabetic nephropathy. Sci
Rep 2018;8:12738.

16. Yuan J, Watanabe M, Suliman M, et al. Serum hepatocyte growth factor is associated with truncal fat mass and increased mortality in chronic kidney disease stage 5 patients with protein-energy wasting. Nephrol Dial Transplant 2015;30:274-282.

17. Kahl A, du Bois A, Harter P, et al. Prognostic value of the age-adjusted Charlson Comorbidity Index (ACCI) on short- and longterm outcome in patients with advanced primary epithelial ovarian cancer. Ann Surg Oncol 2017;24:3692-3699.

18. Lin JX, Huang YQ, Xie JW, et al. Age-adjusted Charlson Comorbidity Index (ACCI) is a significant factor for predicting survival after radical gastrectomy in patients with gastric cancer. $B M C$ Surg 2019;19:53.

19. An JN, Li L, Lee J, et al. Urinary cMet as a prognostic marker in immunoglobulin A nephropathy. J Cell Mol Med 2020;24:1115811169.

20. Koch JP, Aebersold DM, Zimmer Y, Medová M. MET targeting: time for a rematch. Oncogene 2020;39:2845-2862.

21. Nagano T, Mori-Kudo I, Kawamura T, Taiji M, Noguchi H. Pre- or post-treatment with hepatocyte growth factor prevents glycerol-induced acute renal failure. Ren Fail 2004;26:5-11.

22. Zhou D, Tan RJ, Lin L, Zhou L, Liu Y. Activation of hepatocyte growth factor receptor, c-met, in renal tubules is required for renoprotection after acute kidney injury. Kidney Int 2013;84:509520.

23. An JN, Li L, Lee J, et al. cMet agonistic antibody attenuates apoptosis in ischaemia-reperfusion-induced kidney injury. J Cell Mol Med 2020;24:5640-5651.

24. Mizuno S, Matsumoto K, Nakamura T. Hepatocyte growth factor suppresses interstitial fibrosis in a mouse model of obstructive nephropathy. Kidney Int 2001;59:1304-1314.

25. Llovet JM, Peña CE, Lathia CD, et al. Plasma biomarkers as predictors of outcome in patients with advanced hepatocellular carcinoma. Clin Cancer Res 2012;18:2290-2300.

26. Peng F, Liang C, Chang W, et al. Prognostic significance of plasma hepatocyte growth factor in sepsis. J Intensive Care Med 2021 Feb 22 [Epub]. DOI: 10.1177/0885066621993423.

27. Barisione G, Fabbi M, Gino A, et al. Potential role of soluble c-Met as a new candidate biomarker of metastatic uveal melanoma. JAMA Ophthalmol 2015;133:1013-1021.

28. Hass R, Jennek S, Yang Y, Friedrich K. c-Met expression and activity in urogenital cancers: novel aspects of signal transduction and medical implications. Cell Commun Signal 2017;15:10.

29. Kim JH, Jang HJ, Kim HS, Kim BJ, Park SH. Prognostic impact of high c-Met expression in ovarian cancer: a meta-analysis. J Can- 
cer 2018;9:3427-3434.

30. Coxon A, Rex K, Meyer S, et al. Soluble c-Met receptors inhibit phosphorylation of c-Met and growth of hepatocyte growth factor: c-Met-dependent tumors in animal models. Mol Cancer Ther 2009;8:1119-1125.

31. Libetta C, Esposito P, Martinelli C, et al. Hepatocyte growth factor (HGF) and hemodialysis: physiopathology and clinical implications. Clin Exp Nephrol 2016;20:371-378.

32. Vaidya VS, Waikar SS, Ferguson MA, et al. Urinary biomarkers for sensitive and specific detection of acute kidney injury in humans. Clin Transl Sci 2008;1:200-208.

33. Vargas GA, Hoeflich A, Jehle PM. Hepatocyte growth factor in renal failure: promise and reality. Kidney Int 2000;57:1426-1436.

34. Gaião SM, Paiva JA. Biomarkers of renal recovery after acute kidney injury. Rev Bras Ter Intensiva 2017;29:373-381.

35. Fliser D, Novak J, Thongboonkerd V, et al. Advances in urinary proteome analysis and biomarker discovery. J Am Soc Nephrol 2007;18:1057-1071.

36. Rifai N, Gillette MA, Carr SA. Protein biomarker discovery and validation: the long and uncertain path to clinical utility. Nat Biotechnol 2006;24:971-983.

37. Salgia R. MET in lung cancer: biomarker selection based on scientific rationale. Mol Cancer Ther 2017;16:555-565.

38. Rhee H, Jang KS, Shin MJ, et al. Use of multifrequency bioimpedance analysis in male patients with acute kidney injury who are undergoing continuous veno-venous hemodiafiltration. PLoS One 2015;10:e133199.

39. Park KH, Shin JH, Hwang JH, Kim SH. Utility of volume assessment using bioelectrical impedance analysis in critically ill patients receiving continuous renal replacement therapy: a prospective observational study. Korean J Crit Care Med 2017; 32:256-264. 